\title{
Quality of life after salvage surgery for extensively recurrent head and neck cancer patients: a prospective, observational, real-world study
}

\section{Lirui Zhang}

Beijing Stomatological Hospital

Huan Liu

Beijing Stomatological Hospital

Bo Li

Beijing Stomatological Hospital

Jinzhong Li

Beijing Stomatological Hospital

Hao Wang

Beijing Stomatological Hospital

\section{Bin Yang}

Beijing Stomatological Hospital

Lizheng Qin

Beijing Stomatological Hospital

\section{Zhengxue Han}

Beijing Stomatological Hospital

Zhien Feng ( $\sim$ jyfzhen@126.com )

Beijing Stomatological Hospital https://orcid.org/0000-0003-1952-6734

\section{Research Article}

Keywords: Salvage surgery, quality of life, QLQ-H\&N35, UW-QOL

Posted Date: January 26th, 2022

DOI: https://doi.org/10.21203/rs.3.rs-1211340/v1

License: (c) (1) This work is licensed under a Creative Commons Attribution 4.0 International License. Read Full License 


\section{Abstract \\ Purpose}

Multiple studies on salvage surgery (SS) implied the efficacy but the estimate of quality of life (QOL) after SS was poorly analyzed. Our study aimed to examine the quality of life before and after salvage surgery using the validated instruments QLQ-H\&N35 and UW-QOL and investigate the value of salvage surgery by quantifying survival outcomes.

\section{Materials and methods}

Among 21 patients, preoperative and postoperative QOL were accessed using QLQ-H\&N35 and UW-QOL questionnaire. Patients' demographic characteristics, history of treatment, recurrent tumor characteristics, salvage surgery details and follow-up date were abstracted from our prospective database. Statistical analysis methods included Wilcoxon signed-rank test, descriptive analysis and Kaplan-Meier method.

\section{Results}

The overall quality of life was significantly improved after salvage surgery. Pain was significantly relieved whilst diminished functions were hinted with significant differences in UW-QOL domains swallowing, chewing, speech, shoulder, taste, saliva and in QLQ-H\&N35 domains swallowing, speech, social eating, senses, dry mouth, sticky saliva as $\mathrm{p} \otimes 0.05$. The median disease-free survival of whole 21 patients was 5 months (range 0-73) and the median overall survival was 13 months (range 1-73).

\section{Conclusions}

The quality of life after salvage surgery for extensively recurrent head and neck cancer is acceptable. Despite the dysfunctions such as dysphagia and dry mouth, it does relieve the pain and improve the short-term overall QOL of patients. Further studies on quality of life before and after salvage surgery in HNSCC are obviously needed. In the future, postoperative functional recovery training also needs attention during follow up.

\section{Introduction}

The morbidity and mortality of head and neck cancers increased gradually, with over 830000 new cases and nearly 430000 deaths globally in 2018.[1] In head and neck region, squamous cell carcinomas (HNSCC) takes a proportion of more than $90 \%$ [2] and surgery with adjuvant chemoradiotherapy is the most common treatment utilized in head and neck malignant tumors.[3, 4]. Nevertheless, recurrence is still inescapable with rate of $25 \%-50 \%,[5]$ especially for advanced stage cancer (Stage III or IV).[6] Patients with recurrence are faced with less options[7]. The complications caused by previous radiotherapy and 
chemotherapy will also have adverse effect on the therapy.[8-10] In addition, resection of widely spread tumors in head and neck region always leads to appearance changing, dysfunction of swallowing, chewing and others, which profoundly impact their quality of life (QOL). Accordingly, extensively recurrent head and neck cancers raise the treatment challenges both in therapy selection and implementation for surgical team.

The term "salvage surgery" (SS) was firstly came up with in 1965, describing surgical treatment after failure of radiotherapy. It is currently identified as a final attempt for residual and recurrent tumors after definitive treatment, including surgical treatment.[11] Multiple studies on SS implied the efficacy[12] with five-year overall survival(OS) and disease-free survival(DFS) rates of $42 \%$ and $47 \%$ respectively and it has been advocated as the last curative option for recurrent advanced head and neck cancer[13-15]. Although the oncological outcomes of SS showed a substantial improvement, the estimate of quality of life before and after salvage surgery was poorly analyzed. The European Organization for Research and Treatment of Cancer (EORTC) questionnaire and University of Washington Quality of Life (UW-QOL) questionnaire were two most frequently used questionnaires globally measuring QOL of patients with head and neck cancer.[16]

In this study, we tried to examine the quality of life before and after salvage surgery for patients with extensively recurrent head and neck cancer, using the validated instruments QLQ-H\&N35 and UW-QOL. A secondary aim is to investigate the value of salvage surgery by quantifying survival outcomes.

\section{Materials And Methods}

\section{Study sample}

The data of this study originated from POROMS, a Prospective, Observational, Real-world Oral Malignant Tumors Study (clinicaltrials.gov identifier: NCT02395367). This database was established in January 1 , 2015 , based on the department of oral maxillofacial head and neck oncology, Beijing Stomatological Hospital. It covered demographic and clinical characteristics, medical history, postoperative complications and pre- and post- treatment QOL, etc. In this study, the initial study sample included all treated patients with oncologic malignancy from January 1, 2015 to January 31, 2021. Amidst the mentioned group, we selected eligible patients using the following criteria: (1) patients received resection surgery plus reconstruction with latissimus dorsi flaps or pectoralis major myocutaneous flaps; (2) patients with a tumor recurred more than once (including locoregional recurrence and secondary primary tumor); (3) patients with a tumor invading vital structures (internal carotid artery, skull base, pterygoid plate, masticatory space, parapharyngeal space and orbit); (4) patients provided an informed consent. Patients who lost to follow up and refused to participate were excluded from study. (Figure.1) This study was approved by the Beijing Stomatological Hospital ethics committee and conducted with the informed consent of patients.

\section{Instruments}


Pre- and corresponding post- quality of life were measured using QLQ-H\&N35 (version 3.0) and UW-QOL (version 4.0). EORTC Questionnaires has been well accepted and widely used to evaluate the quality of life of cancer patients.[17-19] It contains a general questionnaire, QLQ-Core-30 and a specific module, QLQ-H\&N35, which evaluates the common areas and specific parts of cancer patients, respectively. QLQH\&N35 is composed of 7 multi-item scales assessing pain, problems with swallowing, senses (taste and smell), speech, social eating, social contact and sexuality, and 11 single items assessing problems with teeth, opening the mouth, dry mouth, sticky saliva, coughing, feeling ill, as well as use of analgesics, nutritional supplements, feeding tube, weight gain and weight loss. The raw dates were scored on a fourpoint Likert scale ("not at all," "a little," "quite a bit," "very much"), whereas the last five items were in form of yes/no. The scales scores range from 0 to 100, with higher scores suggested severer symptomatology or problems.[17]

Another questionnaire with the same application is UW-QOL questionnaire, which has been come up with by Hassan and Weymuller[20] in 1993. It consists of 12 single question domains, which contains physical function domains with chewing, speech, swallowing, taste, saliva, appearance, and social function domains with anxiety, mood, pain, activity, recreation, shoulder function.[21] In addition, it also has three global questions, one about how patients feel compared to the month before they developed cancer, one about their health-related quality of life during the last 7days and one about their overall quality of life during the last 7days. For UW-QOL, each domain has 3-6 options scored evenly from 0 to 100, with higher scores implied better quality of life.

\section{Date collection and Integrity}

Patients' demographic characteristics (age, gender), history of treatment (whether underwent radiotherapy, chemotherapy and other treatments), recurrent tumor characteristics (pathologic type, invasion of important structure), salvage surgery details (selection of flaps, results of resection margin) and follow-up date (adjuvant therapy, postoperative complications, DFS and OS) were abstracted from the mentioned database.

All patients registered in database fulfilled postoperative quality of life questionnaires by themselves. Postoperative quality of life questionnaires were completed within 3 months after salvage surgery. Shortterm (less than 3 months) follow-up data were collected via subsequent clinical visit. With regard to longterm follow-up date, we encouraged patients return to outpatient clinic for follow-up to ensure the integrity and authenticity of data and telephone follow-up was only used in patients with poor physical condition that cannot come back to the hospital or death.

\section{Statistical analyses}

The descriptive analysis was used to identify the sample characteristics. Wilcoxon Signed Rank Test, nonparametric test for two paired samples, was used to detecting differences between pre- and postQOL. Significance was established as $\mathrm{p} \bowtie 0.05$. Overall survival and disease-free survival were calculated by Kaplan-Meier. All statistical analyses were carried out using statistical software program SPSS version 26.0 . 


\section{Results}

\section{Sample characteristics}

21 patients were eligible for the study with median age of 59 years (range 43-77) at time of surgery. The proportion of men(57\%) is slightly higher than that of women (43\%). The predominant pathologic type was carcinoma (90\%). Patients' characteristics were shown in Table 1. 
Table 1

Demographic and Clinical characteristics

\section{Characteristics}

Age-year

Median

Range

Gender-n (\%)

Male

Female

Previous surgery methods-n (\%)

Resection of primary tumor

Resection of primary tumor + flap reconstruction

Resection of primary tumor + neck dissection

Resection of primary tumor +flap reconstruction + neck dissection

History of radiotherapy-n (\%)

With

Without

History of chemotherapy-n (\%)

With

Without

History of other treatments-n (\%)

With

Without

Pathologic type-n (\%)

Carcinoma

Sarcoma

Chordoma

Invasion of vital structures- $\mathrm{n}(\%)^{a}$
19(90)

$\mathrm{N}=\mathbf{2 1}$

59

43-77

12(57)

9(43)

$6(28)$

$6(28)$

$6(28)$

a There were 11 patients presented invasion of only one structure, 5 patients got two invaded structures and 5 patients got three. 


\begin{tabular}{|c|c|}
\hline Characteristics & $\mathrm{N}=21$ \\
\hline Mastication muscles space and parapharyngeal space & 20(95) \\
\hline Skull base & $6(29)$ \\
\hline Internal carotid artery & $2(10)$ \\
\hline Pterygoid process & $6(29)$ \\
\hline Orbit & $2(10)$ \\
\hline \multicolumn{2}{|l|}{ Selection of flaps-n (\%) } \\
\hline Latissimus dorsi flap & 18(86) \\
\hline Pectoralis major musculocutaneous flap & $3(14)$ \\
\hline \multicolumn{2}{|l|}{ Results of salvage surgery-n (\%) } \\
\hline Negative margin & $12(57)$ \\
\hline Positive margin & $6(29)$ \\
\hline Positive margin $\rightarrow$ negative margin & $3(14)$ \\
\hline \multicolumn{2}{|l|}{ Adjuvant therapy-n (\%) } \\
\hline None & $8(38)$ \\
\hline Radiotherapy & $6(28)$ \\
\hline Chemotherapy & $5(24)$ \\
\hline Radiotherapy + Chemotherapy & $2(10)$ \\
\hline \multicolumn{2}{|l|}{ Complications-n (\%) } \\
\hline With & $3(14.3)$ \\
\hline Maxillofacial edema & 2 \\
\hline Flap crisis & 2 \\
\hline Bone exposure & 1 \\
\hline Pharyngo-cutaneous fistula & 1 \\
\hline Without & $22(88.0)$ \\
\hline
\end{tabular}

\section{Salvage surgery}


In terms of the history of prior treatments, patients received resection of primary tumor with or without neck dissection or reconstruction were all 6(29\%), and the last 3(14\%) patients received resection of primary tumor with both. The number of patients had received radiotherapy or chemotherapy or other treatments were $7(33 \%), 3(14 \%), 2(10 \%)$, respectively.

Mastication muscles space and parapharyngeal space were the most frequently involved structures with $20(95 \%)$ patients, while invasion of skull base involved, internal carotid artery, pterygoid process, orbit were $6(29 \%), 2(10 \%), 6(29 \%), 2(10 \%)$, respectively. There were 11 patients presented invasion of only one structure, 5 patients got two invaded structures and 5 patients got three. Most patients chose latissimus dorsi flap (86\%). In view of organ preservation and avoiding of fatal complications, approximately $29 \%$ patients can't got negative margin, which we called cancer-free rection margin.

The operation process of a typical salvage surgical case is shown in Figure 2. This is a case of a huge recurrent SCC of the maxillofacial region which the lesions involved the oral cavity, skin of the zygomatic face, the outer orbital, sinuses and deep facial area (Figure 2A-C). And radiographic images presented tumor encroached maxillary and mandibular bone, maxillary sinus and orbital floor (Figure 2D-E). The case underwent an extensive resection including maxilla, mandibular ramus, oral buccal mucosa and gingiva, and Zygomatic facial skin and lateral orbital bone wall (Figure 2F). And then, the huge defect was reconstructed with double-skin paddle free latissimus dorsi flaps and titanium mesh implantation of right orbit after all margins were negative (Figure 2G-J). Finally, the case obtained a primary cure, received a satisfactory quality of life, and the tumor was never recurrence followed up for 1 year.

Learned from the follow-up notes, $6(29 \%)$ and $5(24 \%)$ patients underwent concomitant radiotherapy and chemotherapy, respectively, $2(10 \%)$ patients received both and $8(38 \%)$ did not receive any adjuvant therapy. The incidence of complications seems low with only $3(14 \%)$ patients, manifested as maxillofacial edema, flap crisis, bone exposure and pharyngo-cutaneous fistula.

\section{Quality of life measured by UW-QOL}

The scores of UW-QOL before and after salvage surgery were shown in Table 2. The mean scores of overall quality of life prior to salvage surgery was $30.95(95 \% \mathrm{Cl}, 25.99-35.92)$, while that after salvage surgery was $84.52(95 \% \mathrm{Cl}, 76.10-92.95)$. It revealed significant differences ( $\mathrm{p} \otimes 0.001)$ with better quality of life after salvage surgery. Among 12 functional domains, the lowest preoperative scores were found in pain(57.14) and lowest postoperative scores were chew(30.95). As for comparative analyses of UW-QOL scores between pre- and post- salvage surgery, significant differences were found in 7 function domains which were pain $(p=0.021)$, swallowing $(p \otimes 0.001)$, chewing $(p=0.003)$, speech $(p=0.004)$, shoulder $(p=0.004)$, taste $(p \otimes 0.001)$, saliva $(p=0.011)$. Among the former items, the domain "pain" achieved better scores which implied the relief after salvage surgery, however, worse scores were found in others hinted the diminished functions. (Figure 3) 
Table 2

Comparison of quality of life between preoperative and postoperative with UW-QOL

UW-QOL
Preoperative

$\operatorname{Mean}(95 \% \mathrm{Cl})$
Postoperative

$\operatorname{Mean}(95 \% \mathrm{Cl})$
Differencevalue*

Mean $(95 \% \mathrm{Cl})$
Pvalue

\section{Functional domains}

\begin{tabular}{|c|c|c|c|c|}
\hline Pain & $\begin{array}{l}57.14(51.88- \\
62.41)\end{array}$ & $\begin{array}{l}\text { 72.62(63.16- } \\
82.08)\end{array}$ & $\begin{array}{l}15.48(2.77- \\
28.19)\end{array}$ & 0.021 \\
\hline Appearance & $\begin{array}{l}\text { 67.86(58.94- } \\
76.78)\end{array}$ & $\begin{array}{l}\text { 69.05(62.91- } \\
75.18)\end{array}$ & $\begin{array}{l}1.19(-11.00- \\
13.38)\end{array}$ & 0.868 \\
\hline Activity & $\begin{array}{l}\text { 75.00(68.77- } \\
81.23)\end{array}$ & $\begin{array}{l}71.43(57.36- \\
85.50)\end{array}$ & $\begin{array}{l}-3.57(-18.96- \\
11.82)\end{array}$ & 0.743 \\
\hline Recreation & $\begin{array}{l}76.19(70.53- \\
81.85)\end{array}$ & $\begin{array}{l}75.00(63.62- \\
86.38)\end{array}$ & $\begin{array}{l}-1.19(-13.90- \\
11.52)\end{array}$ & 0.926 \\
\hline Swallowing & $\begin{array}{l}80.95(72.33- \\
89.58)\end{array}$ & $\begin{array}{l}37.14(24.50- \\
49.79)\end{array}$ & $\begin{array}{l}-43.81(-57.50- \\
-30.12)\end{array}$ & $\varangle 0.001$ \\
\hline Chewing & $\begin{array}{l}59.52(50.37- \\
68.68)\end{array}$ & $\begin{array}{l}30.95(17.53- \\
44.37)\end{array}$ & $\begin{array}{l}-28.57(-43.96- \\
-13.18)\end{array}$ & 0.003 \\
\hline Speech & $\begin{array}{l}75.24(63.23- \\
87.25)\end{array}$ & $\begin{array}{l}40.95(27.61- \\
54.29)\end{array}$ & $\begin{array}{l}-34.26(-52.53- \\
-16.05)\end{array}$ & 0.004 \\
\hline Shoulder & $\begin{array}{l}100.00(100.00- \\
100.00)\end{array}$ & $\begin{array}{l}\text { 73.81(57.43- } \\
90.19)\end{array}$ & $\begin{array}{l}-26.19(-42.70- \\
-9.81)\end{array}$ & 0.004 \\
\hline Taste & $\begin{array}{l}100.00(100.00- \\
100.00)\end{array}$ & $\begin{array}{l}64.29(49.57- \\
79.01)\end{array}$ & $\begin{array}{l}-35.71(-50.43- \\
-20.99)\end{array}$ & $\varangle 0.001$ \\
\hline Saliva & $\begin{array}{l}81.43(74.63- \\
88.22)\end{array}$ & $\begin{array}{l}70.95(65.03- \\
76.87)\end{array}$ & $\begin{array}{l}-10.47(-17.45- \\
-3.50)\end{array}$ & 0.011 \\
\hline Mood & $\begin{array}{l}75.0(69.91- \\
80.09)\end{array}$ & $\begin{array}{l}63.10(48.87- \\
77.32)\end{array}$ & $\begin{array}{l}-11.90(-27.84- \\
4.03)\end{array}$ & 0.136 \\
\hline Anxiety & $\begin{array}{l}74.29(69.39- \\
79.18)\end{array}$ & $\begin{array}{l}70.48(58.31- \\
82.65)\end{array}$ & $\begin{array}{l}-3.81(-17.20- \\
9.58)\end{array}$ & 0.227 \\
\hline
\end{tabular}

\section{Composite domains}

Compared to the month before you developed cancer

Health-related quality of life during the past 7 days

Overall quality of life during the past 7 days

\begin{tabular}{|c|c|c|c|}
\hline $\begin{array}{l}21.43(17.35- \\
25.51)\end{array}$ & $\begin{array}{l}\text { 82.14(71.26- } \\
93.02)\end{array}$ & $\begin{array}{l}60.71(48.47- \\
72.96)\end{array}$ & $\nabla 0.001$ \\
\hline $\begin{array}{l}30.95(25.99- \\
35.92)\end{array}$ & $\begin{array}{l}84.52(76.10- \\
92.95)\end{array}$ & $\begin{array}{l}53.57(43.86- \\
63.28)\end{array}$ & $\nabla 0.001$ \\
\hline $\begin{array}{l}30.95(25.99- \\
35.92)\end{array}$ & $\begin{array}{l}84.52(76.10- \\
92.95)\end{array}$ & $\begin{array}{l}53.57(43.86- \\
63.28)\end{array}$ & $\nabla 0.001$ \\
\hline
\end{tabular}

*Postoperative value minus preoperative value 


\section{Quality of life measured by QLQ-H\&N35}

The results of QLQ-H\&N35 were analogous to UW-QOL and listed in Table 3. Pain (p囚0.001) was significantly relieved whilst dysfunction were found with respect to swallowing ( $p \otimes 0.001$ ), speech $(p=0.006)$, social eating $(p=0.006)$, senses $(p \otimes 0.001)$. Symptoms of dry mouth and sticky saliva were severer with P-value of 0.011 and 0.046 respectively compared to prior to surgery. Higher frequency of use of nutritional supplements $(\mathrm{p} \otimes 0.001)$ and feeding tube $(\mathrm{p}=0.046)$ can also been found, accompany with increase in weight(pष0.001). (Figure 4) 
Table 3

Comparison of quality of life between preoperative and postoperative with EORTC QLQ-H\&N35

\section{QLQ-H\&N35}

Preoperative

Mean $(95 \% \mathrm{Cl})$
Postoperative

$\operatorname{Mean}(95 \% \mathrm{Cl})$
Difference-value*

Mean(95\% Cl)
P. value

\section{Multi-items scales}

Pain

27.78(22.50-

33.05)

Swallowing

7.54(4.17-

10.91)

Senses

$0.00(0.00-0.00)$

15.34(7.27-

23.42)

Speech

Social eating

17.86(11.71-

24.01)

Social contact

$14.68(8.90-$

20.47)

13.33(6.82-19.84)

18.25(11.09-25.41)

$40.21(29.08-51.34)$

30.95(24.37-37.54)

$0.00(0.00-0.00)$

$0.00(0.00-0.00)$
$-20.24(-26.65-$

$-13.83)$

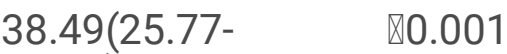

$51.21)$

18.25(11.09-

25.41)

24.87(9.7939.95)

$13.10(4.74-$ 21.46)

$-1.34(-11.28-$

8.58)

$0.00(0.00-0.00) \quad 1.000$

\section{Single-item}

question

\begin{tabular}{|c|c|c|c|c|}
\hline Teeth & $1.59(-1.72-4.90)$ & $1.59(-1.72-4.90)$ & $0.00(-4.80-4.80)$ & 1.000 \\
\hline Opening mouth & $\begin{array}{l}38.10(21.98- \\
54.21)\end{array}$ & 33.33(33.33-33.33) & $\begin{array}{l}-4.76(-20.89- \\
11.35)\end{array}$ & 0.519 \\
\hline Dry mouth & $\begin{array}{l}20.63(13.08- \\
28.18)\end{array}$ & $33.33(25.02-41.64)$ & $\begin{array}{l}12.70(3.75- \\
21.64)\end{array}$ & 0.011 \\
\hline Sticky saliva & $\begin{array}{l}19.05(11.35- \\
26.74)\end{array}$ & $25.40(18.77-32.02)$ & $6.35(0.24-12.45)$ & 0.046 \\
\hline Coughing & $0.00(0.00-0.00)$ & $0.00(0.00-0.00)$ & $0.00(0.00-0.00)$ & 1.000 \\
\hline Felt ill & $\begin{array}{l}31.75(25.92- \\
37.58)\end{array}$ & $38.10(27.06-49.13)$ & $6.35(-2.78-15.48)$ & 0.157 \\
\hline Pain killers & $\begin{array}{l}52.38(29.08- \\
75.68)\end{array}$ & $33.33(11.35-55.32)$ & $\begin{array}{l}-19.05(-58.78- \\
20.69)\end{array}$ & 0.317 \\
\hline $\begin{array}{l}\text { Nutritional } \\
\text { supplements }\end{array}$ & $\begin{array}{l}4.76(-5.17- \\
14.70)\end{array}$ & $\begin{array}{l}100.00(100.00- \\
100.00)\end{array}$ & $\begin{array}{l}95.24(85.30- \\
105.20)\end{array}$ & $\otimes 0.001$ \\
\hline
\end{tabular}

*Postoperative value minus preoperative value 


\begin{tabular}{|c|c|c|c|c|}
\hline \multirow[t]{2}{*}{ QLQ-H\&N35 } & Preoperative & Postoperative & Difference-value* & \multirow[t]{2}{*}{$\begin{array}{l}\mathrm{P}- \\
\text { value }\end{array}$} \\
\hline & Mean(95\%Cl) & Mean(95\%Cl) & Mean(95\%Cl) & \\
\hline Feeding tube & $0.00(0.00-0.00)$ & 19.04(0.73-37.36) & $\begin{array}{l}19.05(0.73- \\
37.36)\end{array}$ & 0.046 \\
\hline Weight loss & $\begin{array}{l}28.58(7.50- \\
49.64)\end{array}$ & $14.28(-2.04-30.61)$ & $\begin{array}{l}-14.29(-40.38- \\
11.81)\end{array}$ & 0.257 \\
\hline Weight gain & $0.00(0.00-0.00)$ & $61.90(39.25-84.56)$ & $\begin{array}{l}61.90(39.25- \\
84.56)\end{array}$ & $\varangle 0.001$ \\
\hline
\end{tabular}

\section{Survival outcomes}

As of January 31, 2021, only 33\% of patients were alive. 2-year and 5-year disease-free survival were $24 \%$ and $5 \%$, respectively, while 2-year and 5 -year overall survival were $52 \%$ and $5 \%$, respectively. The median disease-free survival of whole 21 patients was 5 months (range 0-73) and the median overall survival was 13 months (range 1-73). (Figure 5)

\section{Discussion}

In our study, we considered salvage surgery as the treatment for extensively recurrent head and neck cancer. Demonstrated by the questionnaires' outcomes, the QOL of patients has improved after salvage surgery, despite the declines of some functions. The consequences of UW-QOL were in accordance with QLQ-H\&N35 in the assessment of physical functions and social functions.

Pain was one of the common symptoms of cancer patients, with no exception in head and neck cancers and it had noticeable impact on QOL. Studies on understanding priorities of patients with HNSCC showed the most concerning of cure, survival and avoiding pain,[22] hinted that it's crucial to improve QOL and enhance their confidence for treatment modalities and life expectancy, aside from prolonging the survival time. Salvage surgery, in the premise of balance with function, can remove the invasion and compression on nerve. Hence, the performance of SS significantly contributed to the relief of severe pain. The compression of tumor also leads to the change of appearance. Better scores were found after SS while with no significant difference. We thought it was caused by the small sample size.

Dysphagia occurred frequently after treatment for HNSCC because of the gross destruction of organs vital for swallowing (e.g. tongue, larynx, mouth floor and pharyngeal wall).[23, 24] The same consequence was found in our study that swallowing decreased significantly with lower scores, in which 17 of 21 patients suffered partly or totally dysphagia and all of them got one or all of these structures involved. The dysfunction of chewing and speech, with significant difference, interrelated with the former anatomical structures as well, combined with masticatory space and jaws. Disorders of these functions provided a powerful push to malnutrition, weight loss and other severe health problem, which adversely 
impacted the QOL of carcinoma patients. In other words, the site of tumors was relevant to the QOL. Meanwhile, problems of swallowing and chewing, together with tongue destruction and use of feeding tube probably displeased the taste experience, which may decline the QOL slightly.

It was found that problems with shoulder received significant difference. By inspecting the scores date against patients' records, we found out that the four patients with the worst shoulder scores had terrible general condition. As for those patients with decreased scores, two of them underwent accessory nerve snipped. We hypothesized the performance of neck dissection as one possible reason, which might bring about the excision or destruction of accessory nerve. The other reason might be the preparation of latissimus dorsi flaps or pectoralis major myocutaneous flaps because the truncated tissue contributed to the dysfunction.

Dry mouth is a significant manifestation of salivary gland dysfunction, confirmed as the side effect after radiotherapy[25] and reduced salivary production or sticky saliva was associated with the decline in QOL. [26] In our study, significant differences of sticky saliva and dry mouth can be found between preoperative-scores and postoperative-scores with a tendency to become more severe. Surprisingly, we found patients received radiotherapy accompanied with worse function of salivary gland and their postoperative scores of domain "sticky saliva" differed significantly to those in patients have not received radiotherapy ( $\mathrm{x} \otimes 0.001)$. We speculated that radiotherapy might be a possible cause, combined with excision of parotid gland and submandibular gland.

Several previous studies indicated the high rates of complications after SS in head and neck cancer ranged from $23-67 \%$ and pharyngo-cutaneous fistula was the most common one.[14, 27] Complications after SS in our study, however, were seldom with only 3 patients, manifested in maxillofacial edema, flap crisis, bone exposure and pharyngo-cutaneous fistula. We considered the inconsistent result was relevant to small sample size and recall bias with some postoperative follow-up information were provided by family members of patient and sorrow of relative's loss might influence the reminiscence.

We tentatively estimated survival time and found that patients underwent salvage surgery achieved 2year overall and disease-free survival of $52 \%$ and $24 \%$ respectively and sharply reduced 5 -year overall and disease-free survival of $5 \%$ in both. The latter is lower than previous study outcomes.

Limitations do exist in this study. Limited sample size is worrying due to the rarity of patients. Because of the terrible physical condition of some patients that cannot come back to the hospital, some postoperative follow-up date were obtained through telephone, which undoubtedly increased the inaccuracy of the date. Likewise, criterion for responses to the question differs from each patient due to the subjective character. Conservative Chinese caused missing value of sexuality as well.

\section{Conclusions}

The quality of life after salvage surgery for extensively recurrent head and neck cancer is acceptable. Despite the dysfunctions such as dysphagia and dry mouth, it does relieve the pain and improve the 
short-term overall QOL of patients. Further studies on quality of life before and after salvage surgery in HNSCC are obviously needed. In the future, postoperative functional recovery training also needs attention during follow up.

\section{Declarations}

The patient provided written informed consent.

\section{Conflict of interest}

The authors declare that they have no conflicts of interest to this work.

\section{Acknowledgment}

This article is supported by the Capital's Funds for Health Improvement and Research (CFH2020-2-2143), the National Natural Science Foundation of China (82072984); the Project of Beijing Municipal Education Commission (KM202110025008); and Beijing Science and Technology Commission (Z161100000516201).

\section{References}

1. Cramer, J. D., et al. (2019). Immunotherapy for head and neck cancer: Recent advances and future directions. Oral Oncol, 99, 104460

2. Ruud Kjaer, E. K., et al. (2020). The Impact of Comorbidity on Survival in Patients With Head and Neck Squamous Cell Carcinoma: A Nationwide Case-Control Study Spanning 35 Years. Front Oncol, 10,617184

3. Pignon, J. P., et al. (2000). Chemotherapy added to locoregional treatment for head and neck squamous-cell carcinoma: three meta-analyses of updated individual data. The Lancet, 355, 949955

4. Forastiere, A., et al. (2003). Concurrent Chemotherapy and Radiotherapy for Organ Preservation in Advanced Laryngeal Cancer. ;349:2091-8

5. Ho, A. S., et al. (2014). Decision making in the management of recurrent head and neck cancer. Head Neck, 36, 144-151

6. Haque, S., et al. (2019). High-risk pathological features at the time of salvage surgery predict poor survival after definitive therapy in patients with head and neck squamous cell carcinoma. Oral Oncol, $88,9-15$

7. Saba, N. F., et al. (2018). Salvage surgery for squamous cell carcinoma of the head and neck in the era of immunotherapy: Is it time to clarify our guidelines? Cancer, 124, 4163-4164

8. Kwon, D., et al. (2018). Overcoming wound complications in head and neck salvage surgery. Auris Nasus Larynx, 45, 1135-1142 
9. Esteller, E., et al. (2011). Salvage surgery after locoregional failure in head and neck carcinoma patients treated with chemoradiotherapy. Eur Arch Otorhinolaryngol, 268, 295-301

10. Banaszewski, J., et al. (2019). Primary surgical treatment versus salvage surgery: Results of free flap reconstruction performed in 100 patients with oropharyngeal cancer. Surg Oncol, 28, 174-179

11. Sanabria, A., et al. (2014). Salvage surgery for head and neck cancer: a plea for better definitions. Eur Arch Otorhinolaryngol, 271, 1347-1350

12. Patil, V. M., et al. (2020). Salvage surgery in head and neck cancer: Does it improve outcomes? Eur J Surg Oncol, 46, 1052-1058

13. Elbers, J. B. W., et al. (2019). Salvage surgery for advanced stage head and neck squamous cell carcinoma following radiotherapy or chemoradiation. Eur Arch Otorhinolaryngol, 276, 647-655

14. Hamoir, M., et al. (2018). The Current Role of Salvage Surgery in Recurrent Head and Neck Squamous Cell Carcinoma. Cancers (Basel), 10, 267

15. Tan, H. K., et al. (2010). Salvage surgery after concomitant chemoradiation in head and neck squamous cell carcinomas - stratification for postsalvage survival. Head Neck, 32, 139-147

16. Qin, S. H., et al. (2018). Systematic retrospective study of oral cancer-related quality of life scale. Hua xi kou qiang yi xue za zhi = Huaxi kouqiang yixue zazhi =. West China journal of stomatology, 36, $410-420$

17. Bjordal, K., et al. (1999). Quality of life in head and neck cancer patients: validation of the European Organization for Research and Treatment of Cancer Quality of Life Questionnaire-H\&N35. ;17:100819

18. Bjordal, K., et al. (2000). A 12 country field study of the EORTC QLQ-C30 (version 3.0) and the head and neck cancer specific module (EORTC QLQ-H\&N35) in head and neck patients. ;36:1796-807

19. Aaronson, N. K., et al. (1993). The European Organization for Research and Treatment of Cancer QLQ-C30: a quality-of-life instrument for use in international clinical trials in oncology. ;85:365-76

20. Hassan, S. J., et al. (2010). Assessment of quality of life in head and neck cancer patients. ;15:48596

21. Boyapati, R. P., et al. (2013). Quality of life outcome measures using UW-QOL questionnaire v4 in early oral cancer/squamous cell cancer resections of the tongue and floor of mouth with reconstruction solely using local methods. Br J Oral Maxillofac Surg, 51, 502-507

22. Windon, M. J., et al. (2019). Priorities, concerns, and regret among patients with head and neck cancer. Cancer, 125, 1281-1289

23. Lopez-Jornet, P., et al. (2012). Assessing quality of life in patients with head and neck cancer in Spain by means of EORTC QLQ-C30 and QLQ-H\&N35. J Craniomaxillofac Surg, 40, 614-620

24. Pezdirec, M., et al. (2019). Swallowing disorders after treatment for head and neck cancer. Radiol Oncol, 53, 225-230

25. Riley, P., et al. (2017). Pharmacological interventions for preventing dry mouth and salivary gland dysfunction following radiotherapy. Cochrane Database Syst Rev, 7, CD012744 
26. Winter, C., et al. (2021). Investigation of Changes in Saliva in Radiotherapy-Induced Head Neck Cancer Patients. Int J Environ Res Public Health, 18, 1629

27. Hamoir, M., et al. (2017). Salvage surgery in recurrent head and neck squamous cell carcinoma: Oncologic outcome and predictors of disease free survival. Oral Oncol, 67, 1-9

\section{Figures}

\section{Figure 1}

Flow chart of identification of eligible patients.

\section{Figure 2}

A typical salvage surgery case. A-C. primary tumor outside and inside the mouth; D-E. radiographic images presented tumor encroached maxillary and mandibular bone, maxillary sinus and orbital floor; $F$. excision of tumor led to orbit exposure; $\mathrm{G}-\mathrm{H}$. reconstruction with double-skin paddle free latissimus dorsi flaps and Titanium mesh implantation of right orbit; I-J. immediate postoperative images.

\section{Figure 3}

Mean change from preoperative scores in UW-QOL and I bars indicate $95 \%$ confidence intervals. Asterisks represent significant difference between pre- and post- scores using Wilcoxon Signed Rank Test. Significance was established as $\mathrm{p} \otimes 0.05$.

\section{Figure 4}

Mean change from preoperative scores in H\&N35 and I bars indicate $95 \%$ confidence intervals. Two domains (sexuality and coughing) were omitted because of no change between pre- and post- scores. Asterisks represent significant difference between pre- and post- scores using Wilcoxon Signed Rank Test. Significance was established as $\mathrm{p} \otimes 0.05$. 


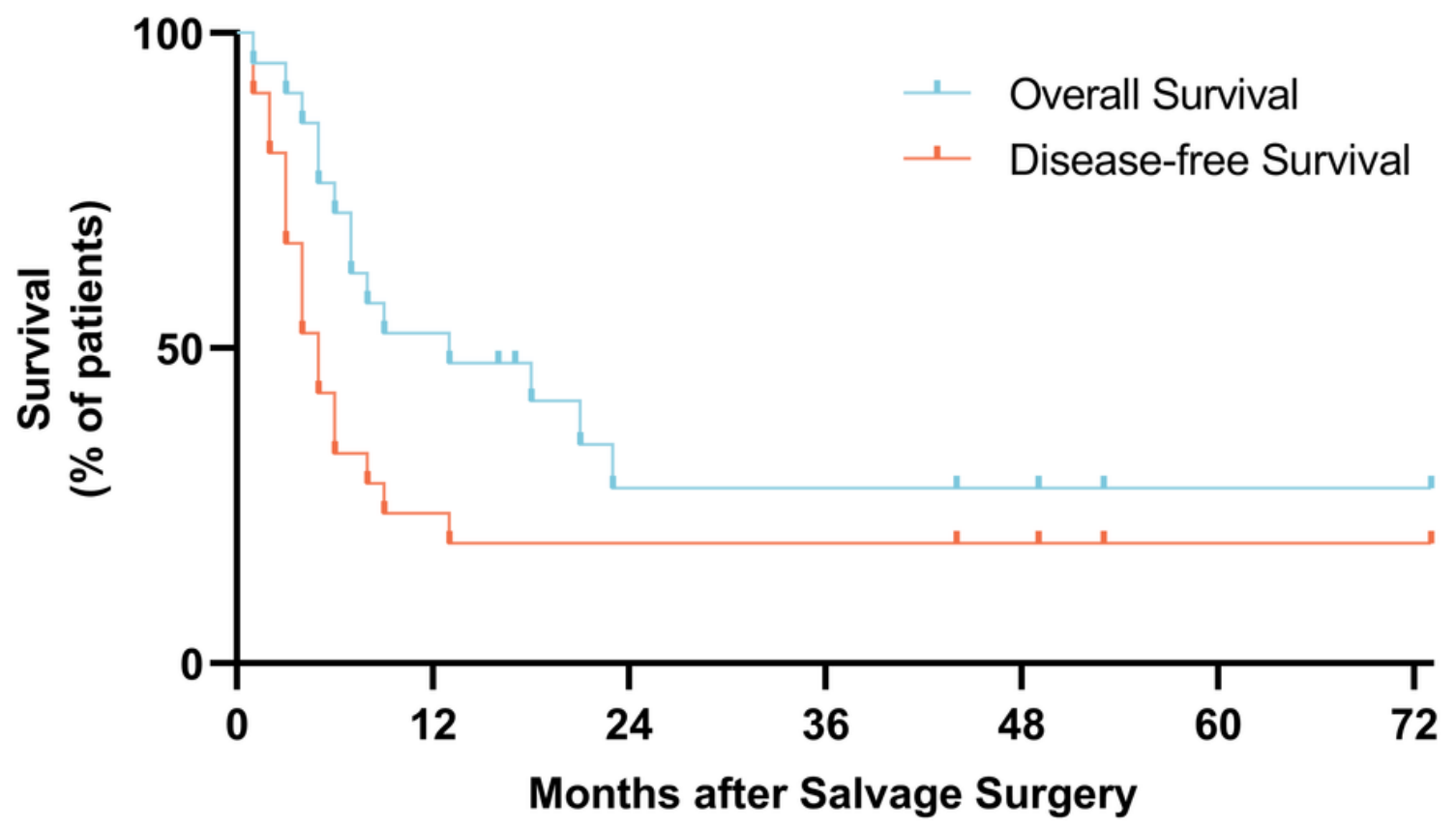

No. at Risk

Overall survival

12

5

4

2

1

Figure 5

Kaplan-Meier estimate of overall survival and disease-free survival of patients after salvage surgery 